\title{
Research and Application Study on Accident-Based 3D Power Station Simulation Technology
}

\author{
Tie Li, Xiaoming Jin, Miao Liü ${ }^{1,}$, Shan Jiang, Xu Zhang, Yuanlai Zhang ${ }^{2, b}$ and \\ Dalu $\mathrm{Li}^{3, \mathrm{C}}$ \\ ${ }^{1}$ Liaoning Electric Power Co., Ltd, State Grid Corporation of China, Shenyang, Liaoning, 110004 \\ 2 Tellhow software Co., Ltd. Nanchang, China, Nanchang, Jiangxi, 330096 \\ ${ }^{3}$ Liaoning Electric Power Co., Ltd, State Grid Corporation of China, Shenyang, Liaoning, 110004 \\ a zengyf1234@163.com, ${ }^{\text {b }}$ unsw01@hotmail.com, ${ }^{\mathrm{c}} 740695129 @ q q . c o m$
}

\begin{abstract}
Keywords: accident-based 3D simulation, 3D images, transformer substation, on-site investigation, electric grid

Abstract: Nowadays, 3D simulation technology is widely used for training electric grid employees in term of simulating transformer substations. However, there is also the need for accident handling and simulation that transitional 3D technologies cannot be applied. This paper introduces an accident-based 3D simulation system that could collect and classify the real-time information of accidents that occur during the power system operation. When any an accident having been detected, the 3D images can be produced automatically, as a result, the personnel who are in charge of scheduling and controls can roam in the 3D power station simulation system and propose a better solution to the accident via on-site investigation, surrounding equipment and station configuration check. With the help of this new technology, emergency solutions could be made immediately after fault occurs. Hence real-time response takes less time than it was before, and the harm to electric grid is greatly reduced.
\end{abstract}

\section{Introduction}

At present, 3D simulation technology is mainly applied to train working personnel from transformer substations in grid's training institutions, concretely to use of simulation of a single substation for learning other than real-time monitoring. Actually to say, that can't be used for accident handling and simulation. CIS system is mainly applied to line operation and maintenance and management, depending on which dispatching operators can carry out offline query, but can't fix accident position and learn about detailed information ${ }^{[1,2]}$. The system proposed in this paper follows the trend and introduces 3D Simulation and GIS systems for stable control and relay protection, depending on which the system can carry out accident simulation and assist dispatching operators in accident analysis and fast solutions.

Nowadays, the fact that the present substations are almost unattended ones decides that the dispatching operators are unable to check substation's internal configuration and equipment status which are super important for accident analysis ${ }^{[3,4]}$. To resolve these problems, applying 3D simulation system can simulate the internal configuration of the substation with troubles and help dispatching operators perform accident analysis and provide solutions as fast as possible. Besides these, 3D simulation systems can be used for accident training, in detail, set a fault artificially, hence the dispatching operators can check the 3D image of the place where the fault occurs, further the substation's internal configuration, as a result, be well trained for better accident analysis and decision-making ${ }^{[5]}$. GIS system can show all $220 \mathrm{KV}$ and above wiring lines and substations in the map, depending on which the dispatching operators can check the wiring lines in the area and substation conditions. When an accident occurs, GIS system is able to fix the accident's position directly, further analyze the diagrams of grids surrounded the accident position, then help to make fast response ${ }^{[6]}$. 


\section{Accident-Based Simulation}

This system is applied to collect and classify the real-time information of accidents that occur during the power system operation, furthermore, connected to 3D Power Station Simulation System and GIS (Geographical information Systems). When any an accident having been detected, the 3D images can be produced automatically, as a result, the personnel who are in charge of scheduling and controls can roam in the 3D power station simulation system and propose a better solution to the accident via on-site investigation, surrounding equipment and station configuration check. When an accident related to circuit failure, the system may fix the position with troubles in GIS and remind the dispatching operators of accident position via flashing lights, then dispatching operators can have a direct view of $220 \mathrm{KV}$ and above grid diagram in the whole area in the map, besides the system can also assist dispatching operators in accident analysis. This system applies GIS Server, 3D Simulation Server, AS Application Server and Data Storage Server.

This system includes two applications as follows:

3D Simulation Module. This module can produce 3D images of the power station, carry out remote operation, give instructions to any equipment and receive its correct response. Meanwhile, if any accident occurs, it can fix the position of the equipment that with troubles automatically, next deduce fault type depending on the preset program, and finally assist to make emergency solution. This whole process can be seen in Fig. 1.

GIS Module. This module works to produce completed grid diagrams in the whole area including 220KV and above wiring lines and position of transformer substation (300 substations and 800 lines). Besides, it can arise an alarm against failures and remind dispatching operators of making real-time response via flashing lights. Also, this is an accident -based system depending on 3D Simulation and GIS system.

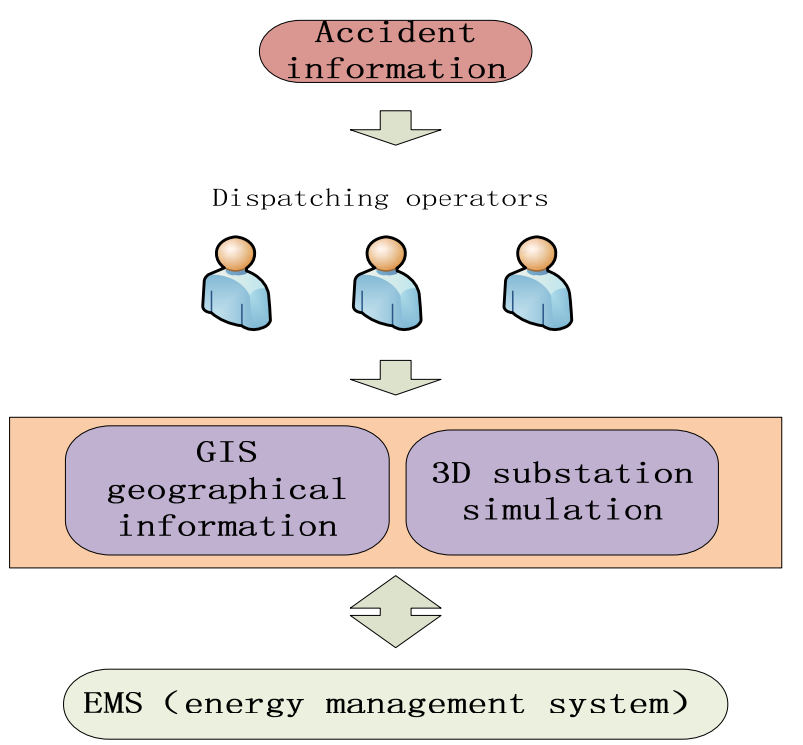

Fig. 1 The Work Flow of Simulation Module

This system combines 3D simulation and GIS system in response of grid faults, whose direct and accurate data can help dispatching operators with accident analysis.

When any accident having been monitored inside a substation, the system can produce the substation's 3D image and connect with EMS system (Grid Operation Information Control Platform), as a result, the accident position can be fixed accurately. The 3D simulation system at the provincial station can indicate the equipment that meets faults automatically and analyze fault types (such as: lightening, fire, floaters, etc.) The dispatching operators can roam within 3D simulation system, have a direct investigation on site, check and examine the substation's configuration and equipment status, finally make a better solution to the accident. When a wiring line with faults having been monitored, the system can fix its position in GIS system, collect the information of faulty phases and pole and tower and remind the dispatching operators of the wiring line with faults via twinkles. Besides these, 
the dispatching operators can directly check the check the geographical conditions of the place where a tripped line occurred on the map (such as mountains, rivers, woods, etc.), and 220KV above grid's diagrams in the whole area and focus more on conditions that multi transmission lines occur on a same tower which may further assist the dispatching operators in accident analysis and handling.

Steps to Implement the System. Dispatching Emergency Command System of Liaoning power Grid has been established upon this method for accidents monitoring and control separately via acquisition terminal, system server and data server. In details, the acquisition terminal can collect a variety of information including equipment information, telemetric data and remote signaling data and then deliver them to system servers. Next data server and system server shall make connection and achieve data interactive ${ }^{[7]}$. Following these data shall be calculated and analyzed, as a result, accidents can be found out timely, so as to malfunction alarms.

The Fault Device Information System:

After new fault information is added to the fault system, the fault device information is sent to the emergency command system via web service, in the form of XML format. When the fault information is received by the emergency command system, D5000 system is checked whether there exists the equipment failure information. This is done by the web service communication way. If there is the information in the library, then check whether there is according equipment failure information locally. If not, then the information is deposited into to the local library.

The Work-Flow Is As Follows:

1. Acquisition terminal works depending on Socket, Web Service and Data Source and others. Hereof Socket long connection mode can receive data from interfaces timely and meet requirements for real-time data collection. The accident acquisition mechanism is complex but smooth. Web Service has characters of cross-platform and cross-system at multiple-interfaces among systems. Data Source can read data from difference databases depending on data transmission method. The work-flow is shown in Fig. 2.

2. The system is fully functional, such as data collection, data analysis, data processing, data filtering and extraction of effective data to assist dispatching department's need in accident handling.

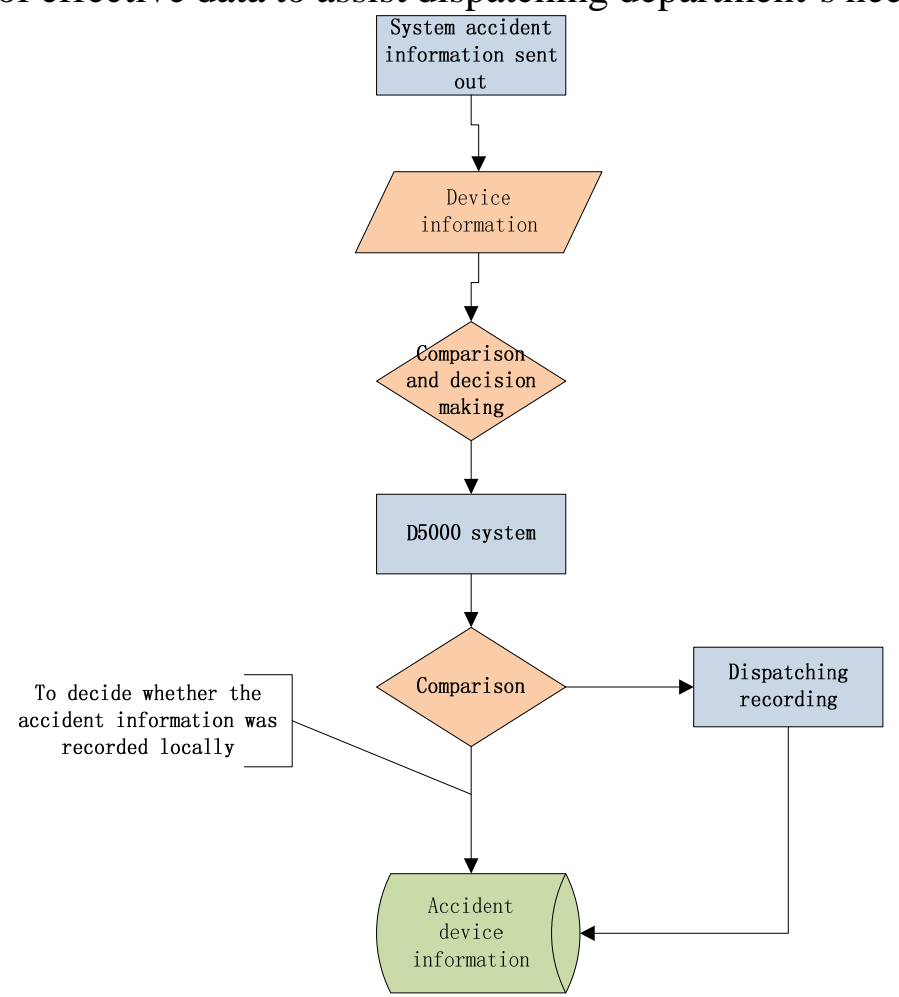

Fig. 2 The Work Flow of Fault Device Information System 
3. Data real-time collection can truly assist the personnel in charge of scheduling in accidents handling. This method is applicable for different dimensions of analysis and statistics, as a result, producing various reports and statements.

Real-Time Status Monitoring:

Obtain the D5000 switch variable information. If the switch position changes and the D5000 system detects the switch changed position. The switch position information is then sent by the web service to emergency command system. After emergency command system gets the message of switching, the dispatching account makes a comparison to see whether scheduling account contains the switch position changed information. And also to judge by the time. If a large account of the schedule contains the information of the switch, it is concluded that the switch is not a fault, but a plan to overhaul the information. The process logic diagram is as followed.

\section{Liaoning Electric Grid 3D Substation Simulation System}

Liaoning electric grid is the most important electric grid in the northeast region in China. Liaoning province is also the energy provider for Beijing and its nearby metro areas. The 3D simulation system has been installed in Liaoning electric grid company and put into operation with the process logic shown in Fig. 3. In particular, Liaoning Grid's 3D Substation Accident Simulation System has the following two modules:

3D Substation Simulation Module which can display the 3D image of any a substation, issue instructions to equipment for operation via Internet and receive correct response that the equipment made against the instructions.

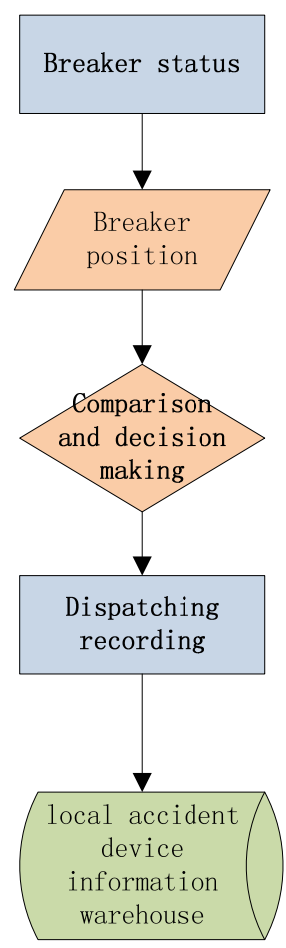

Fig. 3 The Process Logic Diagram in Liaoning Electric Grid

GIS Module can display the positions of $220 \mathrm{kv}$ and above wiring lines and substations in the whole area, produce a completed grid diagrams, send an alarm at fault position via twinkles and remind the dispatching operators of fast and real-time response.

1. Vast information bank constitutes a solid foundation for 3D Substation Simulation and GIS Module, and for modeling and simulation of all equipment components of grid's $220 \mathrm{kV}$ above systems, meanwhile, for better information storage including accident types and wiring lines' surrounding environments. 
2. Real-time accident information of can collected and stored in underlying databases, which can be further delivered to 3D Substation Simulation Module and GIS Module for automatic display of site image or position of the wiring line with faults. For example, if an accident happens to transformer A (code: xj200103), the following diagrams show how a 3D simulation system works. Table 1 shows the accident information of transformer A when the accident occurs. Fig. 4 shows the load on transformer A before, during and after accident takes place. Fig. 5 shows the ACE analysis of transformer A around accident time (15:45pm). Fig. 6 shows the voltage variations of bus-bars which are connected and located near the transformer.

Table 1 The Accident Information of Transformer A

\begin{tabular}{|l|l|l|l|}
\hline Device name & \multicolumn{3}{|l|}{ Transformer A (code: xj200103) } \\
\hline Rated voltage & $220(\mathrm{kV})$ & $\begin{array}{l}\text { Accident } \\
\text { type }\end{array}$ & trip \\
\hline Accident time & $2015-3-20$ & $\begin{array}{l}\text { Time } \\
\text { duration }\end{array}$ & $00 ; 15 ; 45$ \\
\hline $\begin{array}{l}\text { Load } \\
\text { variation(MW } \\
\text { ) }\end{array}$ & 773 & $\begin{array}{l}\text { Accident } \\
\text { level }\end{array}$ & serious \\
\hline $\begin{array}{l}\text { Accident } \\
\text { description }\end{array}$ & $\begin{array}{l}\text { Transformer A \#2 line trip, pre-fault } \\
\text { power flow 773(MW) }\end{array}$ \\
\hline
\end{tabular}

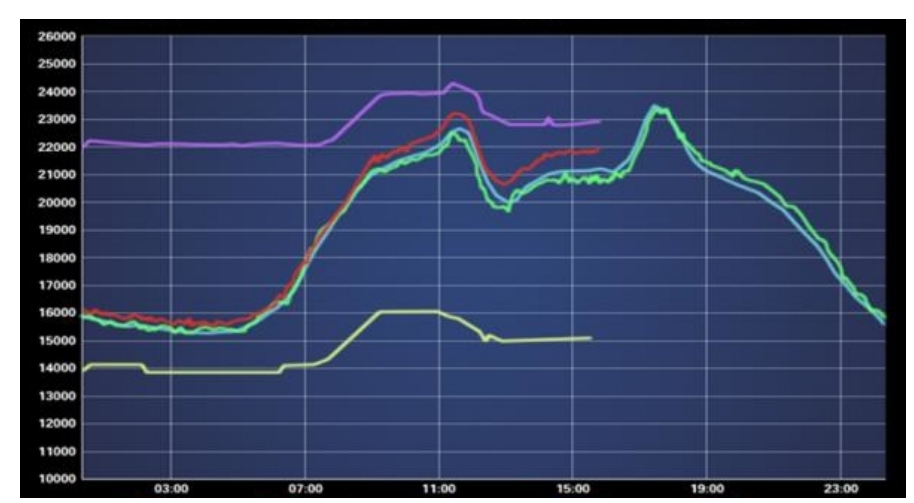

Fig. 4 Load Level of Transformer A

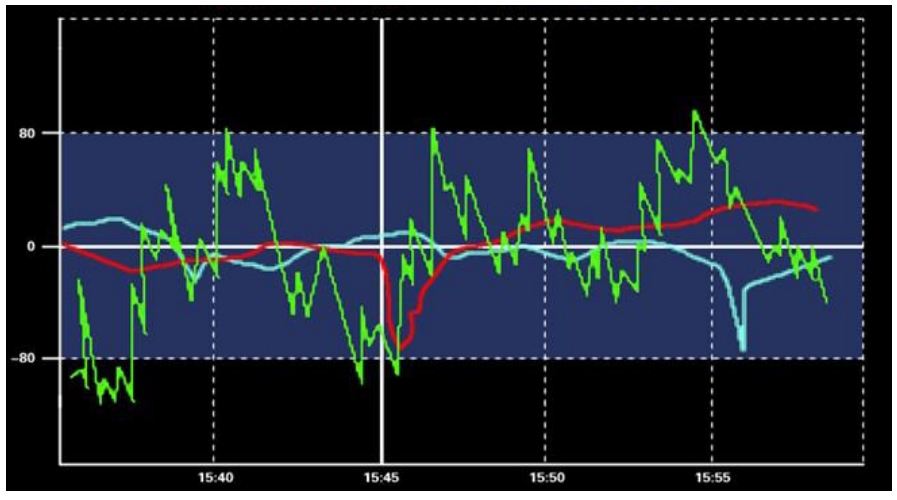

Fig. 5 ACE Analysis of Transformer A

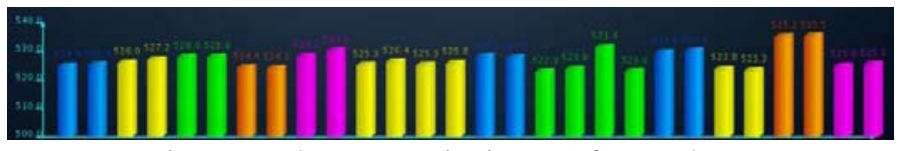

Fig. 6 Voltage Variations of Bus-bars

3. 3D Substation Simulation Module can produce 3D images of the substation with faults via the information delivered, next display type of faults, then issue operational instructions via internet to 
any equipment, finally receive the correct responses that the equipment made against the instructions. Figure 7 shows the 3D simulation of transformer A after the accident occurs.

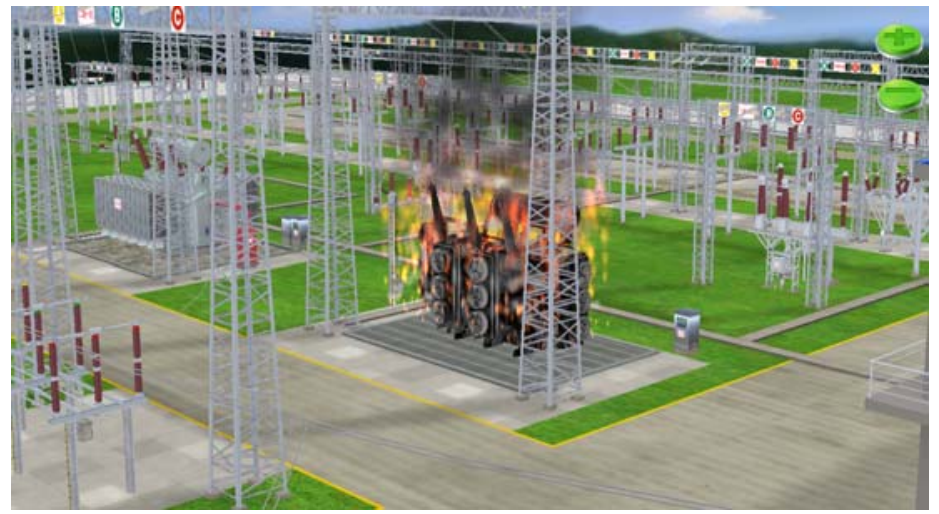

Fig. 7 3D Simulation of Transformer after Accident

4. GIS Module can display the positions of $220 \mathrm{kv}$ and above wiring lines and substations in the whole area via the accident information collected, produce a completed grid diagram, give alarm signals at fault position via twinkles and assist the dispatching operators in fast response.

\section{Conclusions}

The 3D Substation Simulation Module is different from traditional 3D simulation system that can only be used for training. It can display the 3D image of any a substation, issue instructions to equipment for operation via Internet and receive correct response that the equipment made against the instructions. Besides, GIS Module can display the positions of $220 \mathrm{kv}$ and above wiring lines and substations in the whole area. As a result, a completed grid diagrams are produced. An alarm at fault position is sent via twinkles and remind the dispatching operators of fast and real-time response. The applications in Liaoning electric grid network clear shows that, with the help of these technologies, the harm that unexpected faults bring to the electric grid could be greatly reduced; many accidents would be avoided in time.

\section{References}

[1] Overbye T J, Weber J D. Visualizing the Electric Grid [J]. IEEE Spectrum, 2001, 38(2): 52-58.

[2] Overbye T J. Visualizing the Electric Grid for Power Management and Marketing [J]. Electric Light \& Power, 2002, (10): 24-27.

[3] Linder M, Krost G. Advanced Visualization for Power System Operation [J]. Electrical Engineering, 2001, (83): 303-306.

[4] Overbye T J, Weber J D. Visualization of Power System Data [C]. Proceedings of the 33rd Annual Hawaii International Conference on System Sciences, 2000.

[5] Sun Yan, Overbye T J. Visualizations for Power System Contingency Analysis Data [J]. IEEE Trans on Power Systems, 2004, 19(4): 1859-1866.

[6] Junyong Liu, Zhenbo Wei. Design and Application of the Intelligent Dispatch System Based on the Visualization Technology [J]. Distribution \& Utilization, 2010, 27(4): 1- 4. (In Chinese)

[7] Xiaojie Bai, Yuqiang Zhuang, Zimian Qin. Research and Application of Data Visualization in Power Grid Communication Dispatching Field [J]. Telecommunications for Electric Power System, 2013, 34(2):101-105. (In Chinese) 